\title{
Krüppel-like factor 4 modulates the migration and invasion of hepatoma cells by suppressing TIMP-1 and TIMP-2
}

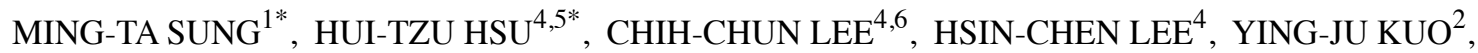 \\ KATE HUA ${ }^{4}$, CHENG-YUAN HSIA ${ }^{3}$ and CHIN-WEN CHI ${ }^{1,4}$ \\ ${ }^{1}$ Department of Medical Research and ${ }^{2}$ Department of Pathology and Laboratory Medicine, and \\ ${ }^{3}$ Department of Surgery, Taipei Veterans General Hospital; ${ }^{4}$ Department and Institute of Pharmacology, School of Medicine, \\ National Yang-Ming University; ${ }^{5}$ Program in Molecular Medicine, National Yang-Ming University and Academia Sinica; \\ ${ }^{6}$ Division of General Surgery, Koo Fundation Sun Yat-Sen Cancer Center, Taipei, Taiwan, R.O.C.
}

Received February 25, 2015; Accepted April 6, 2015

DOI: 10.3892/or.2015.3964

\begin{abstract}
Krüppel-like factor 4 (KLF4) plays important roles in development, stemness and tumorigenesis; however limited information is available on the detailed function of KLF4 in hepatocellular carcinoma (HCC). The objective of the present study was to examine the functional roles of KLF4 in the metastasis of HCC cells. KLF4 was overexpressed and knocked down by lentiviral transduction method in highly metastatic HCC cells. KLF4 overexpression in HCC cells led to inhibition of cell migration and invasion. These inhibitory effects were associated with the upregulation of tissue inhibitors of metalloproteinase (TIMP)-1 and TIMP-2 by KLF4. Treatment with recombinant TIMP-1 decreased the migratory ability of HCC cells. Moreover, myeloperoxidase (MPO)-TIMP-1/TIMP-2 inactivator counteracted the KLF4-induced inhibition of cell migration/invasion. Consistently, KLF4 knockdown in HCC cells downregulated TIMP-1 and TIMP-2 expression, consequently promoting cell migration and invasion. Furthermore, we found that KLF4 regulated E-cadherin and epithelialmesenchymal transition (EMT)-related proteins such as snail, vimentin and Bmil to modulate the cell migration ability. These results together demonstrated for the first time that KLF4 plays an important role in inhibiting the aggressiveness of HCC cells via upregulation of TIMP-1 and TIMP-2.
\end{abstract}

Correspondence to: Dr Cheng-Yuan Hsia, Department of Surgery, Taipei Veterans General Hospital, 201 Shipai Road, Section 2, Taipei 112, Taiwan, R.O.C.

E-mail: cyhsia@vghtpe.gov.tw

Dr Chin-Wen Chi, Department of Medical Research, Taipei Veterans General Hospital, 322 Shipai Road, Section 2, Taipei 112, Taiwan, R.O.C.

E-mail: cwchi@vghtpe.gov.tw

${ }^{*}$ Contributed equally

Key words: KLF4, aggressiveness, TIMP-1, TIMP-2, hepatocellular carcinoma

\section{Introduction}

Hepatocellular carcinoma (HCC) is the fifth most common malignancy associated with a high mortality worldwide (1). To date, surgical resection and liver transplantation are considered the optimal option for the treatment of HCC (2). However, not all HCC patients are suitable for partial hepatectomy or liver transplantation (3). Therefore, to develop more effective therapeutic strategies that directly target key molecules in signaling pathways of tumorigenesis (4) and/or metastasis in HCC is urgently needed.

Krüppel-like factor 4 (KLF4) is mainly found in differentiated epithelial cells of the skin and the gastrointestinal tract (5). KLF4 is a zinc-finger transcription factor that inhibits cell proliferation and maintains the differentiation of epithelial cells by regulating gene expression (6). Moreover, KLF4 expression is also involved in the tumorigenesis and tumor progression in different types of cancer cells (7). KLF4 has been identified as a tumor-suppressor gene, since reduction in KLF4 expression has been reported in esophageal, gastric, colon and lung cancers (8-11). Conversely, KLF4 may also serve as an oncogene since it was found to be increased in oral cancer (12). Thus, these findings suggest that KLF4 has various functions in different type of cancer cells.

Recently, reduction in KLF4 has been found in HCC samples and was significantly correlated with reduced survival time of patients (13). Several reports indicated that KLF4 is involved in the tumor metastasis of HCC and breast cancer cells through suppression of the expression of E-cadherin and epithelial-mesenchymal transition (EMT) inducers such as Slug and Snail (13-15). Using animal model, it has also been demonstrated that KLF4 inhibits the tumorigenic progression of HCC (13). In addition, KLF4 is positively regulated by PPAR $\gamma$ via binding directly to the PPAR response element within the KLF4 promoter in colorectal cancer cells (16). PPAR $\gamma$ agonist, troglitazone, has been found to upregulate KLF4 expression, and this KLF4 induction can be prevented by pretreatment with PPAR $\gamma$ antagonist, GW9662 (16). However, the detailed mechanisms of KLF4 in the modulation of cell motility and invasiveness in hepatoma cells remain unclear. 
Tissue inhibitors of metalloproteinases (TIMPs) control the functions and activities of matrix metalloproteinases (MMPs), which play a crucial role in extracellular matrix degradation involved in tumor cell invasion, metastasis and angiogenesis. For example, increased MMP9 and MMP2 associated with tumor aggressiveness and poor prognosis in HCC (17-20) are regulated by TIMP-1 and TIMP-2, respectively. Additionally, the levels of TIMP-1 and TIMP-2 were also used as prognostic factors for predicting the metastasis of $\operatorname{HCC}(21,22)$. In the present study, we examined the effects of KLF4 on HCC cell migration and invasion. In addition, the relationship between KLF4 and TIMP-1/TIMP-2 was also investigated.

\section{Materials and methods}

Cell culture and reagents. Four HCC cell lines (HepG2, Hep3B, HA22T/VGH and Mahlavu) and HEK293T were maintained in Dulbecco's modified Eagle's medium (DMEM; Invitrogen, Carlsbad, CA, USA) supplemented with $10 \%$ fetal bovine serum (FBS; HyClone, Logan, UT, USA), $0.1 \mathrm{mM}$ non-essential amino acids, $2 \mathrm{mM}$ L-glutamine and $1 \%$ penicillin/streptomycin in a humidified atmosphere containing $5 \%$ $\mathrm{CO}_{2}$ at $37^{\circ} \mathrm{C}$. Rosiglitazone, troglitazone and GW9662 were obtained from Cayman Chemical (Ann Arbor, MI, USA). Recombinant TIMP-1 protein and myeloperoxidase (MPO) were purchased from Enzo Life Sciences (Plymouth Meeting, PA, USA) and Millipore (Temecula, CA, USA), respectively.

Overexpression and knockdown of KLF4. KLF4 was amplified from human cDNA using PCR method with primers: 5'-GTCTAGAGCCACCATGGCTGTCAGCGACGCGCTG-3' and 5'-GGGATCCTTAAAAATGCCTCTTCATG-3'. KLF4 was cloned into the lentiviral expression vector $\mathrm{pLV}-\mathrm{EF} 1 \alpha-$ MCS-IRES-Bsd (pLV-Bsd; Biosettia, San Diego, CA, USA) at the BamHI and NheI restriction enzyme sites. Construct pLVBsd-KLF4 was confirmed by DNA sequencing. The lentiviral vector pLVO.1-shKLF4 used for KLF4 knockdown was purchased from RNAi core of Academia Sinica (Taiwan). The oligonucleotide targeting to human KLF4 was 5'-CTGGAC TTTATTCTCTCCAAT-3'. The lentiviruses were generated by co-transfecting HEK293T cells with the lentiviral expression vectors (pLV-Bsd, pLV-Bsd-KLF4 or pLVO.1-shKLF4) and packaging plasmids (pCMVAR8.91 and pCMV-VSV-G) using T-Pro NTR II (Ji-Feng Biotechnology, Taipei, Taiwan). Supernatants containing the lentivirus were collected $72 \mathrm{~h}$ after transfection. HCC cells were infected with lentivirus in the presence of $8 \mu \mathrm{g} / \mathrm{ml}$ Polybrene (Sigma, St. Louis, MO, USA). KLF4-overexpressing and KLF4-knockdown HCC cells were selected by blastidin $\mathrm{S}$ and puromycin (Sigma), respectively.

Proliferation assay. The tetrazolium salt 3-[4,5-dimethylthiazol-2-yl]-2,5-diphenyltetrazolium bromide (MTT) assay was used to determine the effects of KLF4 overexpression on cell growth. Lenti-Ctrl, Lenti-KLF4, shLuc and shKLF4 cells were seeded at a density of 5,000 cells/well in 96-well plates and incubated for 24, 48 and $72 \mathrm{~h}$. MTT reagent (Sigma-Aldrich) was added into each well of the plates and incubated for another $2 \mathrm{~h}$ at $37^{\circ} \mathrm{C}$. After incubation, formazan crystals were dissolved in dimethylsulfoxide (DMSO), and then optical absorbance at
Table I. DNA sequence of the primers for real-time PCR analysis.

\begin{tabular}{lll}
\hline Gene & & \\
\hline E-cadherin & F & TGA AGG TGA CAG AGC CTC TGG AT \\
& R & TGG GTG AAT TCG GGC TTG TT \\
KLF4 & F & CGA ACC CAC ACA GGT GAG AA \\
& R & TAC GGT AGT GCC TGG TCA GTT C \\
Vimentin & F & CCT TGA ACG CAA AGT GGA ATC \\
& R & GAC ATG CTG TTC CTG AAT CTG AG \\
Bmi1 & F & ACA TCC GAA GCC ACA CGC TGC \\
& R & CGC AGG TTG GAG CGG TCA GC \\
Snail & F & ACA TCC GAA GCC ACA CGC TGC \\
& R & CGC AGG TTG GAG CGG TCA GC \\
MMP2 & F & TTG ACG GTA AGG ACG GAC TC \\
& R & ACT TGC AGT ACT CCC CAT CG \\
MMP9 & F & TTG ACA GCG ACA AGA AGT GG \\
& R & CCC TCA GTG AAG CGG TAC AT \\
TIMP-1 & F & AAG GCT CTG AAA AGG GCT TC \\
& R & GAA AGA TGG GAG TGG GAA CA \\
TIMP-2 & F & CCA AGC AGG AGT TTC TCG AC \\
& R & GAC CCA TGG GAT GAG TGT TT \\
& F & TGG CAT TGC CGA CAG GAT \\
& R & GCT CAG GAG GAG CAATGA TCT \\
& &
\end{tabular}

KLF4, Krüppel-like factor 4; MMP, matrix metalloproteinase; TIMP, tissue inhibitors of metalloproteinases.

a wavelength of $570 \mathrm{~nm}$ was measured by a microplate reader (SpectraMax 250; Molecular Devices, Sunnyvale, CA, USA).

Wound healing, Transwell migration and Transwell invasion assays. For the wound-healing assay, the cells were seeded into each well of the culture insert (Ibidi GmbH, Martinsried, Germany) and incubated overnight. After cells achieved a confluent layer, the culture insert was gently removed. Bright field images were captured after $14 \mathrm{~h}$ using an inverted microscope. The changes in wound area were quantified by ImageJ software.

Cell migration and invasiveness were examined by Transwell polycarbonate membrane inserts (Corning, Corning, NY, USA) and Matrigel basement membrane matrix invasion assay (BD Biosciences, Bedford, MA, USA) according to the manufacturer's instructions and Yang et al (23). Five contiguous fields of cells were counted, and the results were quantified by processing the images using ImageJ software. Data were normalized to the control and represent the average fold-change $\pm \mathrm{SD}$ from three independent experiments.

Real-time quantitative-polymerase chain reaction ( $R T-q P C R)$ analysis. The target genes were amplified with specific primer sets (Table I) and detected by SYBR PCR Master Mix (Applied Biosystems) using ABI PRISM 7900 (Applied Biosystems, 

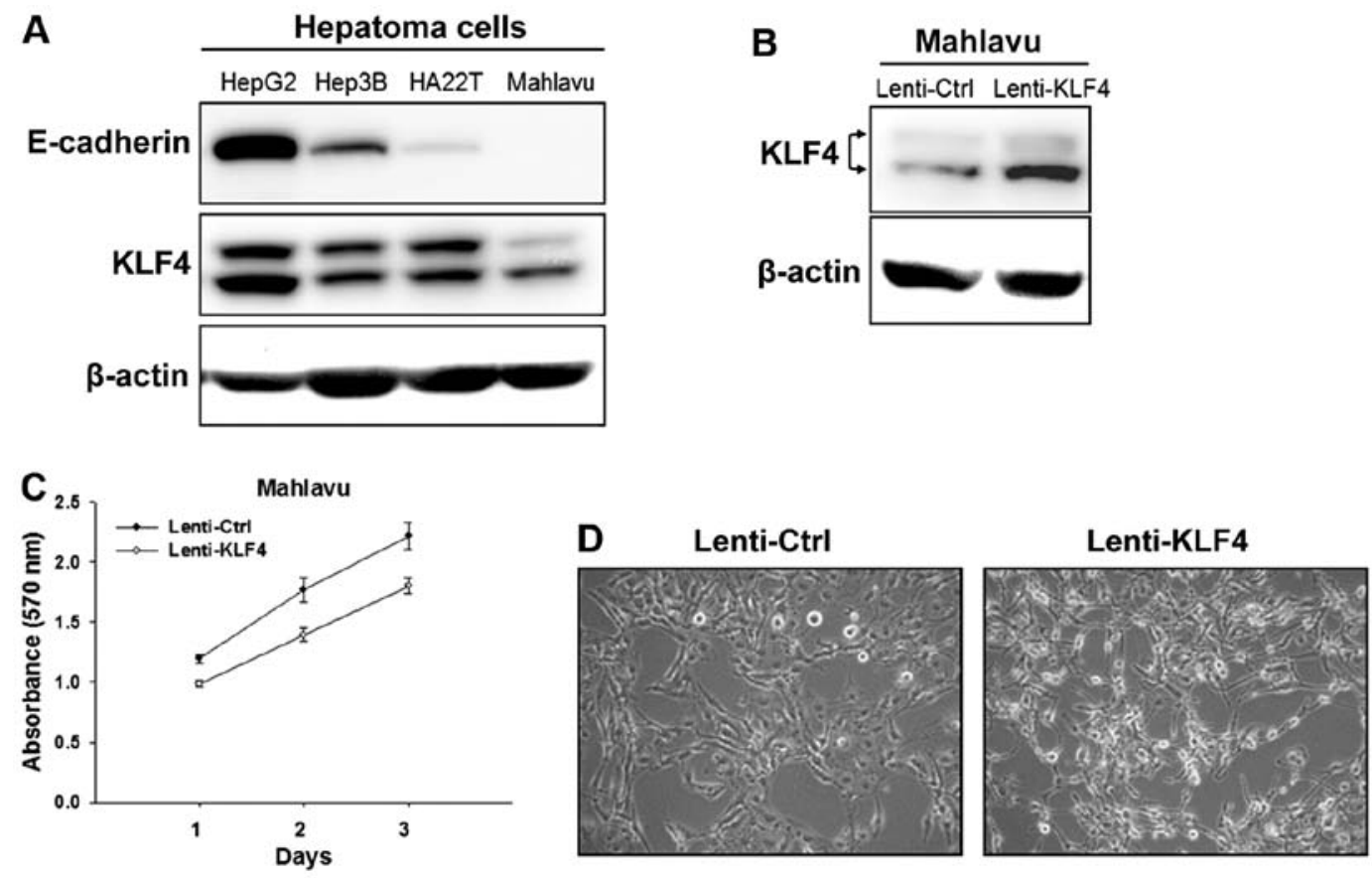

Figure 1. Endogenous expression of KLF4 and E-cadherin in HCC cell lines, and the effects of KLF4 overexpression on cell growth and morphology. (A) Western blot analysis was used to detect the expression of KLF4 and E-cadherin in HepG2, Hep3B, HA22T/VGH and Mahlavu HCC cells. (B) Western blot analysis was used to detect the expression of KLF4 in Lenti-Ctrl vs. KLF4-overexpressing cells. $\beta$-actin was used as a loading control. (C) Cell growth was measured by MTT assay with optical density at $570 \mathrm{~nm}$. Data are expressed as mean \pm SD of eight replicates and are representative from three independent experiments. (D) Inverted light microscopy (magnification, x100) was used to observe the cell morphology. KLF4, Krüppel-like factor 4.

Carlsbad, CA, USA). The alterations in gene expression were obtained using the $\Delta \Delta \mathrm{Ct}$ method in which all samples were first normalized to the level of $\beta$-actin in each sample. Relative normalized units were then compared between the control and KLF4-overexpressing cells.

Western blot analysis. Proteins were separated on $10 \%$ SDS-PAGE and then transferred onto a nitrocellulose membrane. After blocking the membrane in Tris-buffered saline, $0.1 \%$ Tween-20 (TBST) buffer with 5\% skim milk, primary antibodies: E-cadherin, Snail (Cell Signaling, Danvers, MA, USA), KLF4 (Santa Cruz Biotechnology, Santa Cruz, CA, USA), vimentin, $\beta$-actin (Sigma), Bmi1 (Upstate Biotechnology, Lake Placid, NY, USA), MMP2, TIMP-1 (Millipore) and TIMP-2 (NeoMarkers, Fremont, CA, USA) were used to probe the proteins on the membrane at $4^{\circ} \mathrm{C}$ overnight. After incubation with the horseradish peroxidase-conjugated secondary antibody, the probed proteins were detected using the enhanced chemiluminescence system (Millipore) according to the manufacturer's instructions.

Enzyme-linked immunosorbent assay (ELISA). The concentrations of TIMP-1 and TIMP-2 in the cell culture supernatants were analyzed using the ELISA kit (R\&D Systems, Minneapolis, MN, USA) according to the manufacturer's instructions.

Statistical analysis. The means and standard deviation (SD) were calculated from three independent experiments. The Student's t-test was used for comparing the means of two treatment groups. Differences were considered to indicate a statistically significant result when $\mathrm{P}<0.05$.

\section{Results}

KLF4 overexpression inhibits the migratory and invasive abilities in the HCC cells. In order to assess the functional role of KLF4 in HCC cells, we examined the expression of KLF4 in 4 HCC cell lines. It was found that HepG2 cells had the highest level of KLF4 and also the highest level of E-cadherin. In contrast, Hep3B and HA22T/VGH cells had lower levels of KLF4, and Mahlavu cells had the lowest level of KLF4 and undetectable E-cadherin (Fig. 1A). We adopted a lentiviral transduction method to overexpress KLF4 in the Mahlavu cells. Cells were infected by lentivirus with pLVBsd-KLF4 (indicated as Lenti-KLF4), and overexpression of KLF4 expression was confirmed by western blot analysis as compared to cells infected by the lentivirus with the pLV-Bsd vector (indicated as Lenti-Ctrl) (Fig. 1B). Cell proliferative ability was examined by the MTT assay, and the results showed that KLF4 overexpression did not inhibit the growth of the Lenti-KLF4 cells (Fig. 1C). However, KLF4 overexpression led to morphological change in the Lenti-KLF4 cells which showed a round shape and aggregated form as compared to the Lenti-Ctrl cells (Fig. 1D).

We next used wound healing, Transwell migration and Transwell invasion assays to evaluate the migratory and invasive abilities of the Lenti-KLF4 cells. In the wound-healing assay, the Lenti-Ctrl cells almost filled the gap after a 14-h culture, while the Lenti-KLF4 cells showed a 30\% decrease in the migratory ability ( $\mathrm{P}<0.001$, Fig. $2 \mathrm{~A})$. Consistent results were obtained using the Transwell migration assay showing that the Lenti-KLF4 cells had a 50\% decrease in the migratory ability $(\mathrm{P}<0.001$, Fig. 2B). In the Transwell invasion assay, a thin layer of commercial Matrigel was used as extracellular 
A
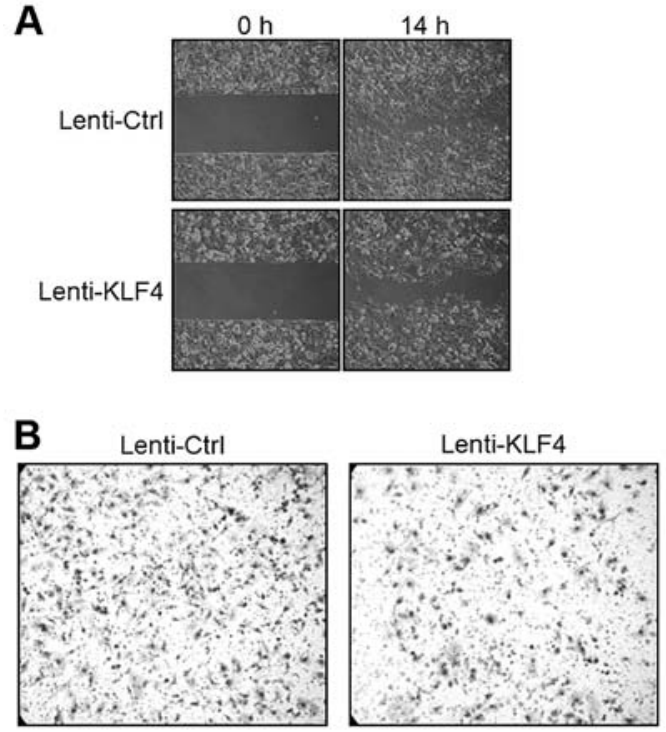

C
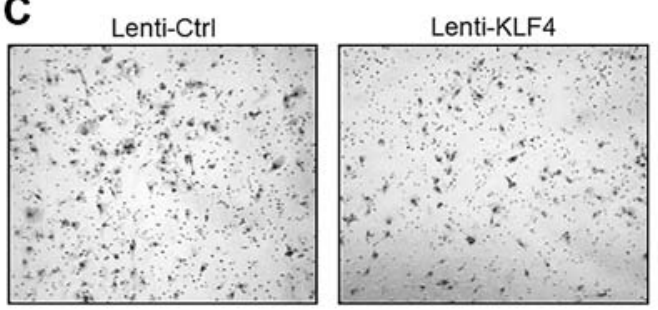
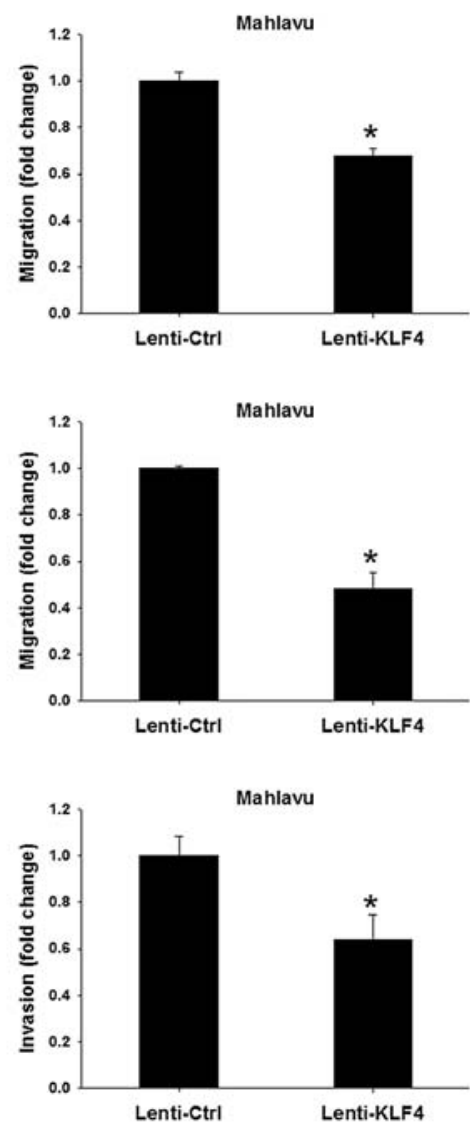

Figure 2. Effects of KLF4 overexpression on the migratory and invasive abilities of HCC cells. Mahlavu cells were infected by a recombinant lentivirus carrying the empty vector or KLF4 (indicated as Lenti-Ctrl or Lenti-KLF4, respectively). (A) Wound-healing, (B) Transwell migration and (C) invasion assays were used to examine the migratory and invasive abilities of the control vs. KLF4-overexpressing cells at the indicated time points. "P<0.001, indicates significantly different from the Lenti-Ctrl cells. KLF4, Krüppel-like factor 4.

matrix. Compared to the Lenti-Ctrl cells, the Lenti-KLF4 cells showed a $35 \%$ decrease in the invasive ability $(\mathrm{P}<0.001$, Fig. 2C). These results clearly demonstrated that KLF4 overexpression significantly suppressed the migratory and invasive abilities but not the growth of HCC cells.

KLF4 overexpression modulates MMPs and TIMPs. To clarify the interaction between KLF4 and proteins involved in cell migration, we examined the effects of KLF4 expression on regulation of the expression of MMPs and TIMPs. The results showed that KLF4 overexpression led to significant upregulation of both the mRNA and protein levels of TIMP-1, and TIMP-2 in the Lenti-KLF4 cells as compared to the LentiCtrl cells. Messenger RNA levels of MMP2 and MMP9 were significantly decreased, yet the protein expression of MMP2 was slightly increased in the Lenti-KLF4 cells (Fig. 3A and B).

To further examine the functional importance of the PPAR $\gamma$ pathway in relation to KLF4 in modulation of TIMP-1 and TIMP-2, we used PPAR $\gamma$ antagonist GW9662 to block the PPAR $\gamma$ pathway. The results showed that GW9662 treatment for $48 \mathrm{~h}$ inhibited the expression of KLF4 in both the Lenti-Ctrl and Lenti-KLF4 cells as compared to the untreated control (Fig. 3B). Additionally, the expression levels of MMP2, TIMP-1, and TIMP-2 were also concomitantly downregulated in both the Lenti-Ctrl and Lenti-KLF4 cells after GW9662 treatment for $48 \mathrm{~h}$.
As newly synthesized TIMPs are secreted and function in extracellular space, we used ELISA assay to determine the concentrations of secreted TIMP-1 and TIMP-2 in the cell culture supernatants. Compared to the Lenti-Ctrl cells, KLF4 overexpression upregulated the concentrations of secreted TIMP-1 and TIMP-2 in the Lenti-KLF4 cells (Fig. 3C and D). However, GW9662 treatment for $48 \mathrm{~h}$ had no effect on the levels of secreted TIMP-1 and TIMP-2 in both the Lenti-Ctrl and Lenti-KLF4 cells.

To clarify the role of KLF4-induced TIMP-1/TIMP-2 expression in relation to migration, we found that treatment with recombinant protein TIMP-1 showed markedly decrease in the migratory ability of the Lenti-Ctrl cells as compared to the untreated cells (Fig. 4A). In addition, we used MPO $\left(50 \mathrm{nM}\right.$ treatment at $37^{\circ} \mathrm{C}$ for $60 \mathrm{~min}$ ) to block the activities of TIMP-1/TIMP-2 in the Lenti-KLF4 cells. The results showed that the migratory and invasive abilities of the MPO-treated Lenti-KLF4 cells were significantly increased as compared to the untreated Lenti-KLF4 cells (Fig. 4B and C). These results together clearly indicate that KLF4 modulates the expression of TIMP-1 and TIMP-2, leading to changes in migration and invasion of HCC cells.

KLF4 silencing inhibits TIMP-1 and TIMP-2 while increasing migration and invasion. In order to further validate the importance of KLF4 in the regulation of cell migration and 

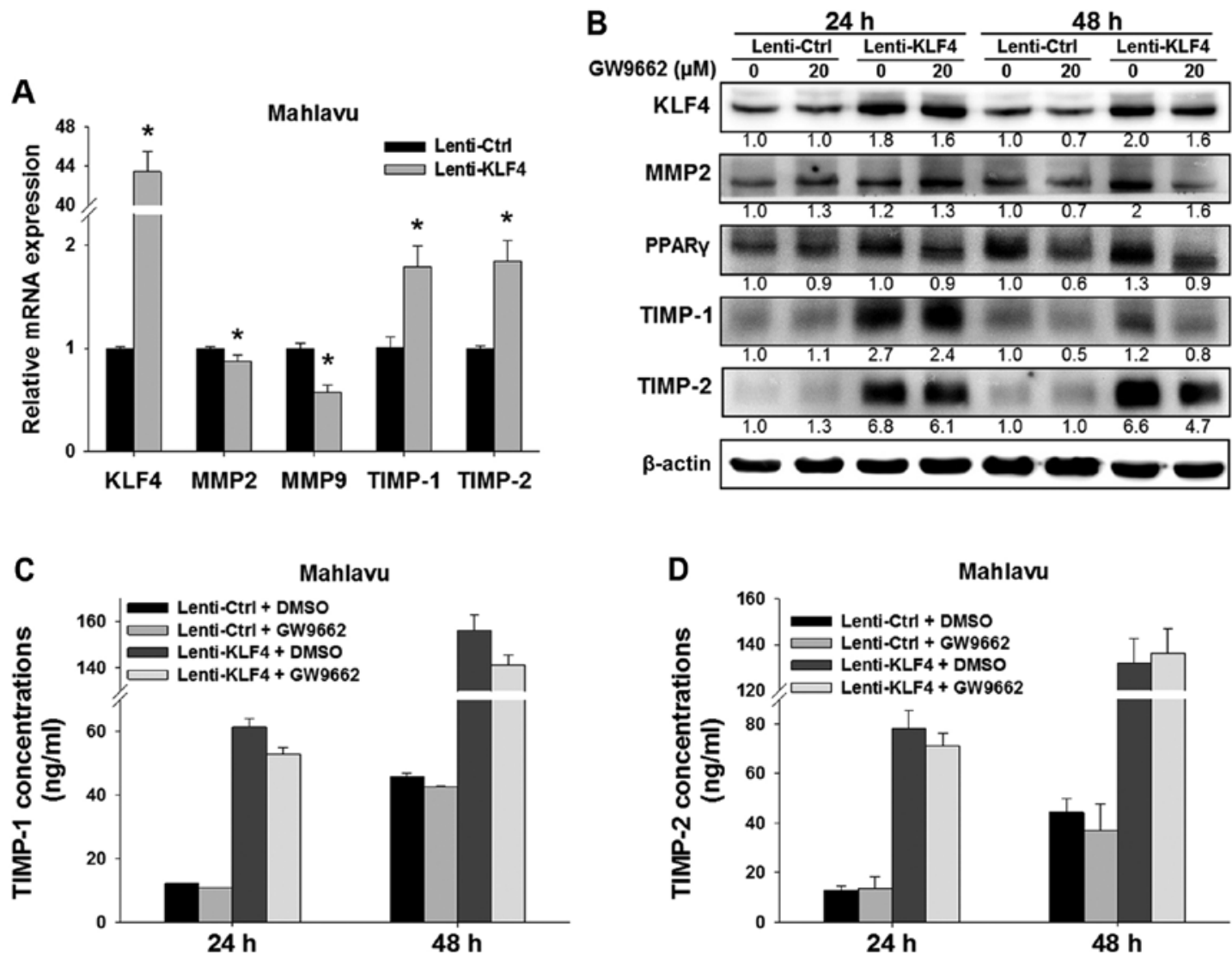

Figure 3. Effects of KLF4 overexpression on MMP2, MMP9, TIMP-1, and TIMP-2 in HCC cells. (A) Real-time qPCR analysis was used to analyze the mRNA levels of KLF4, MMP2, MMP9, TIMP-1 and TIMP-2 in the Lenti-Ctrl vs. Lenti-KLF4 Mahlavu cells. ${ }^{*} \mathrm{P}<0.001$, indicates a significant difference from the Lenti-Ctrl cells. (B) Western blot analysis was used to detect the expression of KLF4, MMP2, PPAR $\gamma$, TIMP-1 and TIMP-2 after treatment with/without GW9662. $\beta$-actin was used as a loading control. Relative fold-changes were indicated as compared to the control at the same time point. (C and D) After treatment with $20 \mu \mathrm{M}$ GW9662, ELISA assay was used to measure the concentrations of secreted TIMP-1 and TIMP-2 in the cell culture supernatants. Representative data are shown from three independent experiments. KLF4, Krüppel-like factor 4; MMP, matrix metalloproteinase.
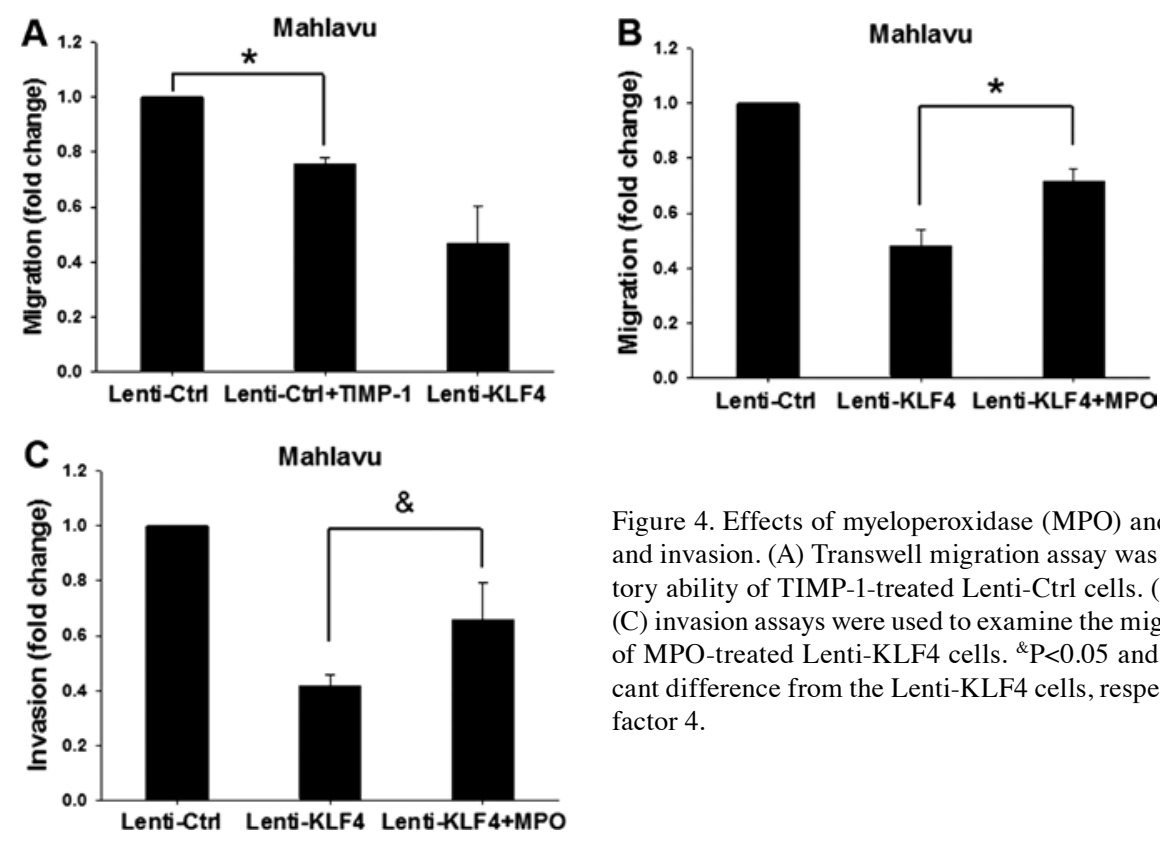

Figure 4. Effects of myeloperoxidase (MPO) and TIMP-1 on cell migration and invasion. (A) Transwell migration assay was used to examine the migratory ability of TIMP-1-treated Lenti-Ctrl cells. (B) Transwell migration and (C) invasion assays were used to examine the migratory and invasive abilities of MPO-treated Lenti-KLF4 cells. ${ }^{\circledR} \mathrm{P}<0.05$ and ${ }^{*} \mathrm{P}<0.001$, indicates significant difference from the Lenti-KLF4 cells, respectively. KLF4, Krüppel-like factor 4 .

invasion, we used lentiviral vector-mediated shRNA to create KLF4-knockdown HA22T/VGH cells (indicated as shKLF4). Compared to the control (indicated as shLuc), the mRNA and protein levels of KLF4 were significantly decreased in the shKLF4 cells (Fig. 5A and B). KLF4 knockdown led to significant upregulation of MMP2 and MMP9 but 
A

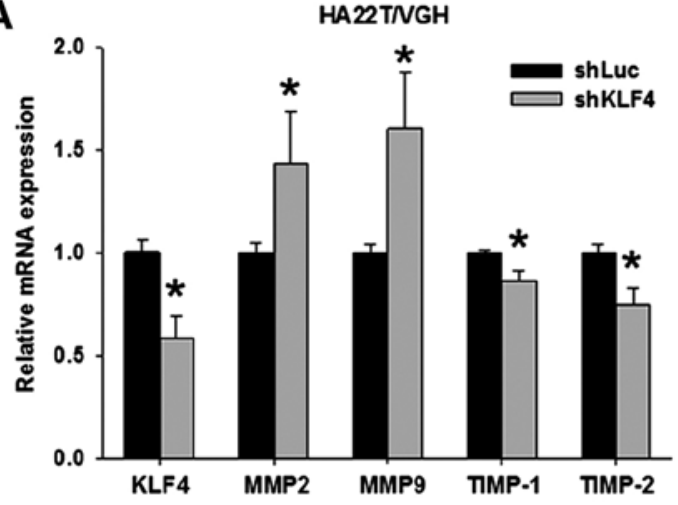

B

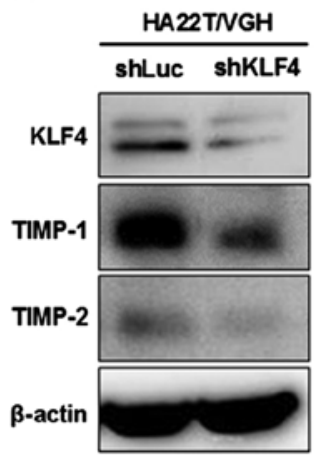

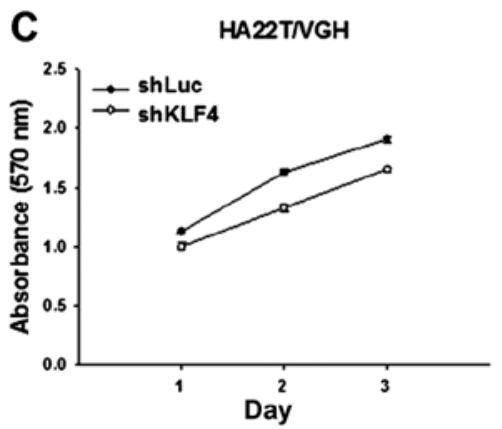
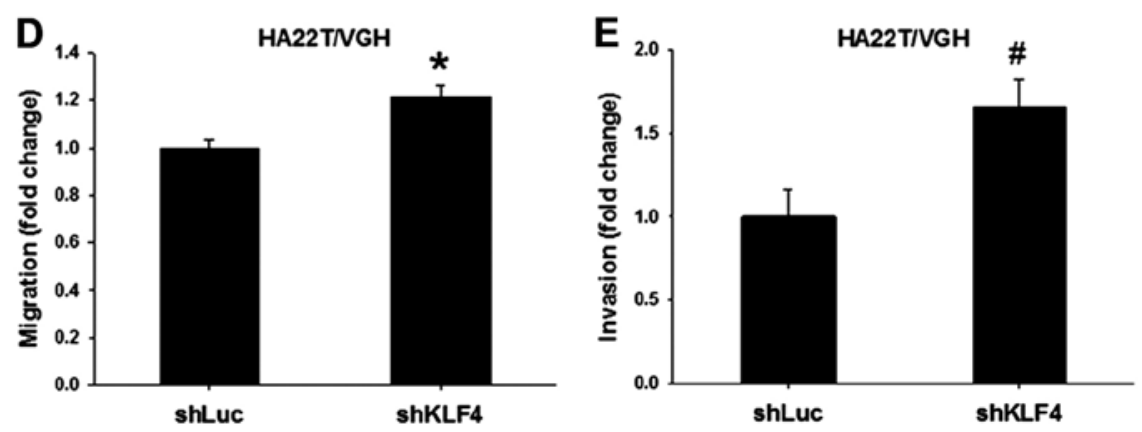

Figure 5. Effects of KLF4 knockdown on cell migration, invasion and the related protein expression in HA22T/VGH cells. (A) Real-time qPCR analysis was used to analyze the mRNA levels of KLF4, MMP2, MMP9, TIMP-1 and TIMP-2 in the control (shLuc) vs.the KLF4-knockdown (shKLF4) cells. * $<0.001$, indicates significant difference from the shLuc cells. (B) Western blot analysis was used to detect the expression of KLF4, TIMP-1 and TIMP-2. $\beta$-actin was used as a loading control. (C) Cell growth was measured by MTT assay with optical density at $570 \mathrm{~nm}$. Data are expressed as mean \pm SD of eight replicates and are representative from three independent experiments. (D) Transwell migration and (E) invasion assays were used to examine the migratory or invasive abilities of the shLuc and shKLF4 cells. ${ }^{*} \mathrm{P}<0.001$ and ${ }^{\#} \mathrm{P}<0.01$, indicate significant difference from the shLuc cells. KLF4, Krüppel-like factor 4.

downregulation of TIMP-1 and TIMP-2 expression in the shKLF4 cells (Fig. 5A and B).

We next evaluated the effects of KLF4 knockdown on cell proliferation, migratory and invasive abilities. In the cell proliferation assay, the result showed that KLF4 knockdown did not alter the growth of the shKLF4 cells (Fig. 5C). Compared to the shLuc cells, the migratory and invasive abilities showed a 1.2-fold $(\mathrm{P}<0.001$, Fig. 5D) and 1.7-fold $(\mathrm{P}<0.01$, Fig. 5E) increase in the shKLF4 cells, respectively. These results clearly demonstrated the critical role of KLF4 in cell migration and invasion by regulating TIMP-1 and TIMP-2 expression.

KLF4 regulates $E$-cadherin and EMT-related proteins. As EMT has been reported to be highly associated with cell migration in $\mathrm{HCC}$, we also evaluated the expression levels of several EMT-related proteins. We found that KLF4 overexpression in the Mahlavu cells resulted in increased E-cadherin and decreased Snail mRNA, yet had no effect on the levels of vimentin and Bmil mRNA (Fig. 6A). Western blot results showed that KLF4 overexpression not only increased the expression of E-cadherin but also inhibited the protein expression of vimentin, Snail and Bmil (Fig. 6B). Moreover, KLF4 knockdown in the HA22T/VGH cells led to decreased E-cadherin, while no significant change in the level of Bmil and Snail mRNA was observed (Fig. 6C). Consistent with KLF4 overexpression, KLF4 knockdown resulted in decreased E-cadherin and increased vimentin protein expres- sion (Fig. 6D). Taken together, these results suggest that KLF4 also plays an important role in regulating E-cadherin and EMT-related proteins to modulate cell migration ability.

\section{Discussion}

It has been reported that KLF4 demonstrates functions to inhibit migration and invasion in several types of cancer (14,24-26); however, the detailed molecular mechanisms in HCC remain unclear. The present study showed that KLF4 overexpression inhibited the migratory and invasive abilities of highly metastatic Mahlavu cells with elevated expression levels of TIMP-1/TIMP-2 (Figs. 2 and 3). Knockdown of KLF4 in the HA22T/VGH cells by shRNA also correlated with increased migration/invasion and reduced TIMP-1/TIMP-2 levels (Fig. 5). Using TIMP inactivator MPO (27), the effects of KLF4 on migration/invasion were partially blocked, suggesting that KLF4 can inhibit cell migration and invasion via upregulation of TIMP-1/TIMP-2 expression (Fig. 4B and C). It has been reported that inhibition of TIMP-1 enhanced the migration of microvascular endothelial cells (28); TIMP-2 overexpression also significantly inhibited migration/invasion of ras-transformed breast epithelial cells (29). It is notable that the expression levels of MMP2, TIMP-1 and TIMP-2 were increased simultaneously in the Lenti-KLF4 cells; however, the increased fold-change of TIMP-1 and TIMP-2 was higher than that of MMP2 (Fig. 3B), consequently resulting in migration/invasion inhibition (Fig. 2). Consistent with our findings, 
A

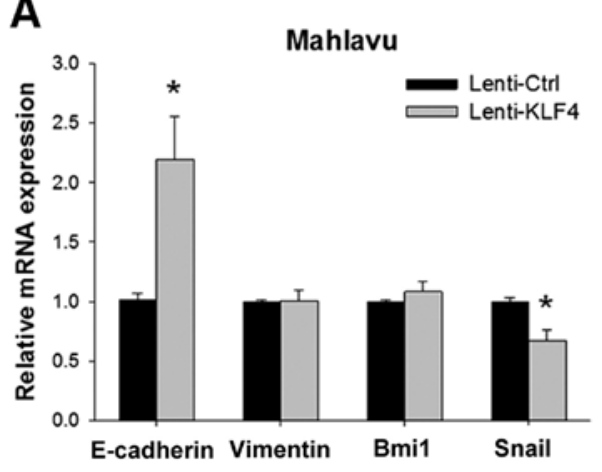

C

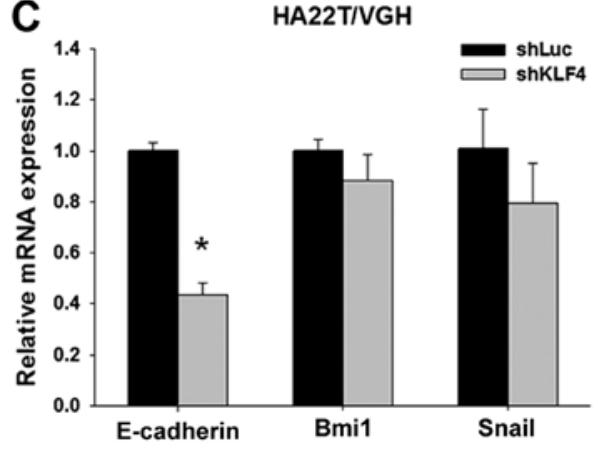

B
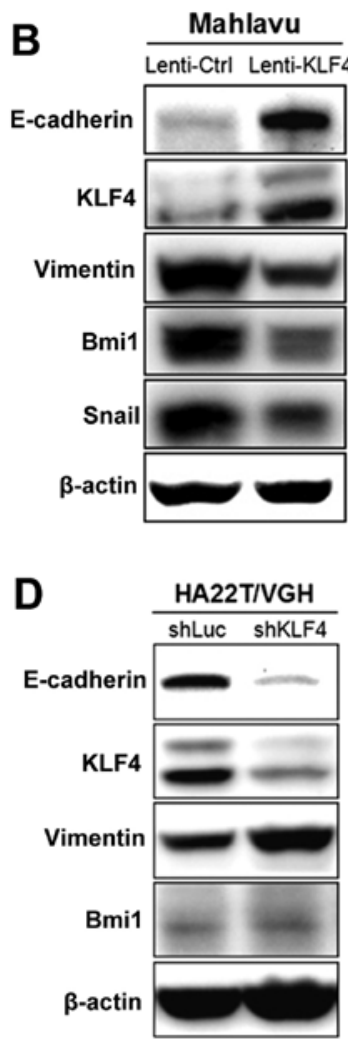

Figure 6. Effects of KLF4 on the expression of EMT-related proteins in the Mahlavu and HA22T/VGH cells. (A and C) Real-time qPCR analysis and (B and D) western blot analysis were used to detect the expression of E-cadherin, KLF4, vimentin, Bmi1 and Snail in the Lenti-Ctrl vs. Lenti-KLF4 Mahlavu cells [or in control (shLuc) vs. KLF4-knockdown (shKLF4) HA22T/VGH cells]. $\beta$-actin was used as a loading control. ${ }^{*}<0.001$ indicate significant difference from the (A) Lenti-Ctrl and (C) shLuc cells, respectively. KLF4, Krüppel-like factor 4; EMT, epithelial-mesenchymal transition.

Wang et al also indicated that KLF4 directly regulates TIMP-2 expression and inhibits migration/invasion in prostate cancer cells (30). Taken together, we demonstrated for the first time that KLF4 suppresses HCC cell migration/invasion through upregulation of TIMP-1 and TIMP-2 expression.

KLF4 has also been reported to inhibit EMT through regulation of E-cadherin expression in breast cancer cells (14). EMT, a process defined by acquisition of mesenchymal phenotype with reduced cell adhesion and increased mobility, plays a key role not only in development but also in malignant tumor progression and metastasis (31). Reduction or loss of E-cadherin expression is considered as an early and critical step to disrupt intercellular contacts and induce the EMT process (32). Moreover, in various types of cancers, the expression of E-cadherin was obviously repressed through the direct binding of transcription factors Snail or Bmil on the E-cadherin promoter $(33,34)$. Li et al found that overexpressed KLF4 induced E-cadherin and reduced Snail in HepG2 and SK-Hep1 cells (13). Consistent with our findings, our results showed that KLF4 overexpression led to an increase in E-cadherin and concomitant decrease in Snail and Bmil in Mahlavu cells (Fig. 6B). On the other hand, it has been reported that TIMP-2 upregulates E-cadherin expression in lung cancer cells (35). Thus, it is most likely that KLF4 not only directly upregulates E-cadherin expression through the transcription level but also indirectly induces TIMP-2 to increase the expression of E-cadherin in HCC cells. These data suggest that KLF4-induced TIMP-1 and TIMP-2 may be the most important factors to regulate HCC migration/invasion in HCC cells.

Vimentin, an intermediate filament, is a marker of mesenchymal cells and has been linked to aggressive tumors. It has been reported that increased vimentin is significantly correlated with the metastasis of HCC (36). Reduction in vimentin by the PPAR $\gamma$ antagonist GW9662 inhibited the migration and invasion of HCC cells (37). This corresponded well with our observation that GW9662 inhibited the expression of KLF4 and TIMP1/TIMP2 (Fig. 3B). Moreover, consistent with our findings, downregulation of vimentin by KLF4 overexpression (Fig. 6B) inhibited the migration and invasion of Mahlavu cells. Moreover, upregulation of vimentin by KLF4 knockdown promoted the migration and invasion of HA22T/VGH HCC cells (Fig. 5). Taken together, our results suggest that the overexpression of vimentin, as a result of loss of KLF4, significantly contributes to HCC progression.

Our findings provide important insights into the mechanism of the KLF4-TIMP-1/TIMP-2 signaling pathway in HCC progression, which inhibits the migration, invasion and metastasis of HCC cells. These unique findings strongly suggest that KLF4 may serve as a potential molecular target in cancer therapy for HCC patients.

\section{Acknowledgements}

We thank Dr Shih-Hwa Chiou for providing the lentiviral expression vector and packaging plasmids. The present 
study was supported by grant NSC100-2320-B-075-003, NSC101-2320-B-075-003, DOH101-TD-C-111-007 and DOH102-TD-C-111-007 from the National Science Council and Ministry of Health and Welfare, Taiwan, respectively.

\section{References}

1. Jemal A, Bray F, Center MM, Ferlay J, Ward E and Forman D: Global cancer statistics. CA Cancer J Clin 61: 69-90, 2011.

2. Tang ZY: Hepatocellular carcinoma - cause, treatment and metastasis. World J Gastroenterol 7: 445-454, 2001.

3. Thomas MB and Abbruzzese JL: Opportunities for targeted therapies in hepatocellular carcinoma. J Clin Oncol 23: 8093-8108, 2005.

4. Whittaker S, Marais R and Zhu AX: The role of signaling pathways in the development and treatment of hepatocellular carcinoma. Oncogene 29: 4989-5005, 2010.

5. Garrett-Sinha LA, Eberspaecher H, Seldin MF and de Crombrugghe B: A gene for a novel zinc-finger protein expressed in differentiated epithelial cells and transiently in certain mesenchymal cells. J Biol Chem 271: 31384-31390, 1996.

6. Chen X, Whitney EM, Gao SY and Yang VW: Transcriptional profiling of Krüppel-like factor 4 reveals a function in cell cycle regulation and epithelial differentiation. J Mol Biol 326: 665-677, 2003.

7. Hsu HT, Sung MT, Lee CC, Chang YF and Chi CW: The role of gut-enriched Krüppel-like factor (GKLF)/KLF4 in gastrointestinal tract-related cancers. J. Cancer Res Pract 27: 191-199, 2011.

8. Zhao W, Hisamuddin IM, Nandan MO, Babbin BA, Lamb NE and Yang VW: Identification of Krüppel-like factor 4 as a potential tumor suppressor gene in colorectal cancer. Oncogene 23: 395-402, 2004.

9. Wei D, Gong W, Kanai M, Schlunk C, Wang L, Yao JC, Wu TT, Huang S and Xie K: Drastic down-regulation of Krüppel-like factor 4 expression is critical in human gastric cancer development and progression. Cancer Res 65: 2746-2754, 2005.

10. Wang N, Liu ZH, Ding F, Wang XQ, Zhou CN and Wu M: Down-regulation of gut-enriched Kruppel-like factor expression in esophageal cancer. World J Gastroenterol 8: 966-970, 2002.

11. Hu W, Hofstetter WL, Li H, Zhou Y, He Y, Pataer A, Wang L, Xie K, Swisher SG and Fang B: Putative tumor-suppressive function of Kruppel-like factor 4 in primary lung carcinoma. Clin Cancer Res 15: 5688-5695, 2009.

12. Foster KW, Ren S, Louro ID, Lobo-Ruppert SM, McKie-Bell P, Grizzle W, Hayes MR, Broker TR, Chow LT and Ruppert JM: Oncogene expression cloning by retroviral transduction of adenovirus E1A-immortalized rat kidney RK3E cells: Transformation of a host with epithelial features by c-MYC and the zinc finger protein GKLF. Cell Growth Differ 10: 423-434, 1999.

13. Li Q, Gao Y, Jia Z, Mishra L, Guo K, Li Z, Le X, Wei D, Huang S and Xie K: Dysregulated Krüppel-like factor 4 and vitamin D receptor signaling contribute to progression of hepatocellular carcinoma. Gastroenterology 143: 799-810.e1-2, 2012.

14. Yori JL, Johnson E, Zhou G, Jain MK and Keri RA: Kruppel-like factor 4 inhibits epithelial-to-mesenchymal transition through regulation of E-cadherin gene expression. J Biol Chem 285: 16854-16863, 2010.

15. Lin ZS, Chu HC, Yen YC, Lewis BC and Chen YW: Krüppel-like factor 4, a tumor suppressor in hepatocellular carcinoma cells reverts epithelial-mesenchymal transition by suppressing slug expression. PLoS One 7: e43593, 2012.

16. Li S, Zhou Q, He H, Zhao Y and Liu Z: Peroxisome proliferatoractivated receptor $\gamma$ agonists induce cell cycle arrest through transcriptional regulation of Kruppel-like factor 4 (KLF4). J Biol Chem 288: 4076-4084, 2013.

17. Bauvois B: New facets of matrix metalloproteinases MMP-2 and MMP-9 as cell surface transducers: Outside-in signaling and relationship to tumor progression. Biochim Biophys Acta 1825: 29-36, 2012.

18. Nart D, Yaman B, Yilmaz F, Zeytunlu M, Karasu Z and Kiliç M: Expression of matrix metalloproteinase-9 in predicting prognosis of hepatocellular carcinoma after liver transplantation. Liver Transpl 16: 621-630, 2010.

19. Cui J, Dong BW, Liang P, Yu XL and Yu DJ: Effect of $c-m y c$, Ki-67, MMP-2 and VEGF expression on prognosis of hepatocellular carcinoma patients undergoing tumor resection. World $\mathbf{J}$ Gastroenterol 10: 1533-1536, 2004.
20. Sakamoto Y, Mafune K, Mori M, Shiraishi T, Imamura H, Mori M, Takayama T and Makuuchi M: Overexpression of MMP-9 correlates with growth of small hepatocellular carcinoma. Int J Oncol 17: 237-243, 2000.

21. Gao ZH, Tretiakova MS, Liu WH, Gong C, Farris PD and Hart J: Association of E-cadherin, matrix metalloproteinases, and tissue inhibitors of metalloproteinases with the progression and metastasis of hepatocellular carcinoma. Mod Pathol 19: 533-540, 2006.

22. Giannelli G, Bergamini C, Marinosci F, Fransvea E, Quaranta M, Lupo L, Schiraldi O and Antonaci S: Clinical role of MMP-2/TIMP-2 imbalance in hepatocellular carcinoma. Int J Cancer 97: 425-431, 2002.

23. Yang MH, Chen CL, Chau GY, Chiou SH, Su CW, Chou TY, Peng WL and Wu JC: Comprehensive analysis of the independent effect of twist and snail in promoting metastasis of hepatocellular carcinoma. Hepatology 50: 1464-1474, 2009.

24. Wei D, Kanai M, Jia Z, Le X and Xie K: Kruppel-like factor 4 induces $p 27^{K i p l}$ expression in and suppresses the growth and metastasis of human pancreatic cancer cells. Cancer Res 68: 4631-4639, 2008.

25. Dang DT, Chen X, Feng J, Torbenson M, Dang LH and Yang VW: Overexpression of Krüppel-like factor 4 in the human colon cancer cell line RKO leads to reduced tumorigenicity. Oncogene 22: 3424-3430, 2003.

26. Li H, Wang J, Xiao W, Xia D, Lang B, Yu G, Guo X, Guan W, Wang Z, Hu Z, et al: Epigenetic alterations of Krüppel-like factor 4 and its tumor suppressor function in renal cell carcinoma. Carcinogenesis 34: 2262-2270, 2013.

27. Wang Y, Rosen H, Madtes DK, Shao B, Martin TR, Heinecke JW and $\mathrm{Fu} \mathrm{X}$ : Myeloperoxidase inactivates TIMP-1 by oxidizing its $\mathrm{N}$-terminal cysteine residue: An oxidative mechanism for regulating proteolysis during inflammation. J Biol Chem 282: 31826-31834, 2007.

28. Reed MJ, Koike T, Sadoun E, Sage EH and Puolakkainen P: Inhibition of TIMP1 enhances angiogenesis in vivo and cell migration in vitro. Microvasc Res 65: 9-17, 2003.

29. Ahn SM, Jeong SJ, Kim YS, Sohn Y and Moon A: Retroviral delivery of TIMP-2 inhibits H-ras-induced migration and invasion in MCF10A human breast epithelial cells. Cancer Lett 207: 49-57, 2004.

30. Wang J, Place RF, Huang V, Wang X, Noonan EJ, Magyar CE, Huang J and Li LC: Prognostic value and function of KLF4 in prostate cancer: RNAa and vector-mediated overexpression identify KLF4 as an inhibitor of tumor cell growth and migration. Cancer Res 70: 10182-10191, 2010.

31. Yang $\mathbf{J}$ and Weinberg RA: Epithelial-mesenchymal transition: At the crossroads of development and tumor metastasis. Dev Cell 14: 818-829, 2008.

32. Onder TT, Gupta PB, Mani SA, Yang J, Lander ES and Weinberg RA: Loss of E-cadherin promotes metastasis via multiple downstream transcriptional pathways. Cancer Res 68: 3645-3654, 2008.

33. Cano A, Pérez-Moreno MA, Rodrigo I, Locascio A, Blanco MJ, del Barrio MG, Portillo F and Nieto MA: The transcription factor snail controls epithelial-mesenchymal transitions by repressing E-cadherin expression. Nat Cell Biol 2: 76-83, 2000.

34. Song LB, Li J, Liao WT, Feng Y, Yu CP, Hu LJ, Kong QL, Xu LH, Zhang X, Liu WL, et al: The polycomb group protein Bmi-1 represses the tumor suppressor PTEN and induces epithelialmesenchymal transition in human nasopharyngeal epithelial cells. J Clin Invest 119: 3626-3636, 2009.

35. Bourboulia D, Han H, Jensen-Taubman S, Gavil N, Isaac B, Wei B, Neckers L and Stetler-Stevenson WG: TIMP-2 modulates cancer cell transcriptional profile and enhances E-cadherin/beta-catenin complex expression in A549 lung cancer cells. Oncotarget 4: 166-176, 2013.

36. Hu L, Lau SH, Tzang CH, Wen JM, Wang W, Xie D, Huang M, Wang Y, Wu MC, Huang JF, et al: Association of Vimentin overexpression and hepatocellular carcinoma metastasis. Oncogene 23: 298-302, 2004.

37. Kim KR, Choi HN, Lee HJ, Baek HA, Park HS, Jang KY, Chung MJ and Moon WS: A peroxisome proliferator-activated receptor $\gamma$ antagonist induces vimentin cleavage and inhibits invasion in high-grade hepatocellular carcinoma. Oncol Rep 18: 825-832, 2007. 